PROCEEDINGS OF THE

AMERICAN MATHEMATICAL SOCIETY

Volume 31, No. 1, January 1972

\title{
METRIC TRANSFORMS AND THE HYPERBOLIC FOUR-POINT PROPERTY
}

\author{
JOSEPH E. VALENTINE AND STANLEY G. WAYMENT
}

\begin{abstract}
The purpose of this paper is to show that any metric space is homeomorphic to a metric space with each quadruple of its points congruently imbeddable in three-dimensional hyperbolic space.
\end{abstract}

1. Introduction. Blumethal, Schoenberg, and others have made extensive investigations concerning metric transforms of euclidean and Hilbert spaces, as well as arbitrary metric spaces. The metric transform of a metric space may be defined as follows $[1, p .130]$.

Definition 1.1. Let $M$ be a metric space and $\phi(x)$ a real valued function defined for every value of $x=p q$, where $p, q$ are points of $M$. A space $\phi(M)$ is the metric transform of $M$ by $\phi$ provided (1) the points of $M$ and $\phi(M)$ are in a one-to-one correspondence, and (2) if points $p^{\prime}, q^{\prime}$ of $\phi(M)$ correspond, respectively, to points $p, q$ of $M$, then $p^{\prime} q^{\prime}=\phi(p q)$.

In this paper the space $\phi(M)$ has the same point-set as $M$, and the biuniform correspondence is the identity; i.e., $\phi(M)$ arises by redefining the distance $p q$ of points $p, q$ to be $\phi(p q)$.

Blumenthal [2, pp. 7-10] has shown that the metric transform $\phi(M)$ of any metric space $M$ by $\phi(x)=x^{\alpha}, 0 \leqq \alpha \leqq \frac{1}{2}$, has the euclidean fourpoint property.

In this paper we will show that the metric transform $\phi(M)$ of any metric space $M$ by $\phi(x)=\cosh ^{-1}\left(x^{2^{\alpha}}+1\right), 0 \leqq \alpha \leqq \frac{1}{2}$, has the hyperbolic four-point property.

In order to facilitate the arguments we introduce the following notation.

The Cayley-Menger determinant of four points $p_{1}, p_{2}, p_{3}, p_{4}$ is defined by

$$
D\left(p_{1}, p_{2}, p_{3}, p_{4}\right)=\left|\begin{array}{cc}
0 & 1 \\
1 & p_{i} p_{j}^{2}
\end{array}\right| \quad(i, j=1,2,3,4)
$$

and the unbordered principal minor of order four of this determinant, $\left|p_{i} p_{j}^{2}\right|(i, j=1,2,3,4)$ is denoted by $C\left(p_{1}, p_{2}, p_{3}, p_{4}\right)$. Since we will be

Received by the editors November 9, 1970.

AMS 1969 subject classifications. Primary 5250, 5040.

Key words and phrases. Cayley-Menger determinant, euclidean four-point property, hyperbolic four-point property, metric transform. 
interested in the determinant obtained from $D\left(p_{1}, p_{2}, p_{3}, p_{4}\right)$, by adding the first row to the second, third, fourth, and fifth rows, respectively, and its unbordered principal minor of order four, we will use the notation

and

$$
D^{\prime}\left(p_{1}, p_{2}, p_{3}, p_{4}\right)=\left|\begin{array}{cc}
0 & 1 \\
1 & p_{i} p_{j}^{2}+1
\end{array}\right|
$$

$$
C^{\prime}\left(p_{1}, p_{2}, p_{3}, p_{4}\right)=\left|p_{i} p_{j}^{2}+1\right| \text {. }
$$

We will denote the symmetric determinant $\left|\cosh p_{i} p_{j}\right|(i, j=1,2,3,4)$ by $\Lambda\left(p_{1}, p_{2}, p_{3}, p_{4}\right)$.

2. The metric transforms. Let $p_{1}, p_{2}, p_{3}$ be any three points of a metric space. Since the function $\phi(x)=\cosh ^{-1}\left(x^{2 \alpha}+1\right)$ is a monotone increasing concave function that vanishes at the origin, it is easily seen that $\phi\left(p_{1} p_{2}\right)+$ $\phi\left(p_{2} p_{3}\right)>\phi\left(p_{1} p_{3}\right)$, for $0 \leqq \alpha \leqq \frac{1}{2}$. Thus, if $p_{1}^{\prime}, p_{2}^{\prime}, p_{3}^{\prime}$ are points with $p_{i}^{\prime} p_{j}^{\prime}=\cosh ^{-1}\left[\left(p_{i} p_{j}\right)^{2 \alpha}+1\right](i, j=1,2,3)$ where $0 \leqq \alpha \leqq \frac{1}{2}$ then $p_{1}^{\prime}, p_{2}^{\prime}, p_{3}^{\prime}$ are not collinear.

THEOREM 2.1. The metric transform $\phi(M)$ of any metric space $M$ by $\phi(x)=\cosh ^{-1}\left(x^{2 \alpha}+1\right), \quad 0 \leqq \alpha \leqq \frac{1}{2}$, has the hyperbolic four-point property.

Proof. First, suppose $\alpha=\frac{1}{2}$. Since $M$ is metric so is $\phi(M)$ and it suffices to show that if $p_{1}^{\prime}, p_{2}^{\prime}, p_{3}^{\prime}, p_{4}^{\prime}$ are four points of $\phi(M)$, then $\Lambda\left(p_{1}^{\prime}, p_{2}^{\prime}, p_{3}^{\prime}, p_{4}^{\prime}\right)=\left|\cosh p_{i}^{\prime} p_{j}^{\prime}\right|=\left|p_{i} p_{j}+1\right| \quad(i, j=1,2,3,4)$ is not positive, see $[3$, p. 224].

Now $\Lambda(\alpha)=\left|\left(p_{i} p_{j}\right)^{2 \alpha}+1\right|(i, j=1,2,3,4)$ is a continuous function of $\alpha$ which is negative for $\alpha=0$. If we suppose $\Lambda\left(\frac{1}{2}\right)>0$, then a number $\alpha_{0}$ exists $0<\alpha_{0}<\frac{1}{2}$, such that $\Lambda\left(\alpha_{0}\right)=0$. It is known that

$$
D\left(p_{1}^{\prime \prime}, p_{2}^{\prime \prime}, p_{3}^{\prime \prime}, p_{4}^{\prime \prime}\right)=\left|\begin{array}{cc}
0 & 1 \\
1 & \left(p_{i} p_{j}\right)^{2 \alpha_{0}}
\end{array}\right| \quad(i, j=1,2,3,4), 0 \leqq \alpha_{0}<\frac{1}{2},
$$

is positive [2, pp. 7-10], and hence $D^{\prime}\left(p_{1}^{\prime \prime}, p_{2}^{\prime \prime}, p_{3}^{\prime \prime}, p_{4}^{\prime \prime}\right)$ is also positive. Denoting by $[2,1]$ the cofactor of the element in the second row and first column of $D^{\prime}\left(p_{1}^{\prime \prime}, p_{2}^{\prime \prime}, p_{3}^{\prime \prime}, p_{4}^{\prime \prime}\right)$ a theorem of determinants gives

$$
\begin{aligned}
C^{\prime}\left(p_{1}^{\prime \prime}, p_{2}^{\prime \prime}, p_{3}^{\prime \prime}, p_{4}^{\prime \prime}\right) \cdot D^{\prime}\left(p_{2}^{\prime \prime}, p_{3}^{\prime \prime}, p_{4}^{\prime \prime}\right)-[2,1]^{2} & =D^{\prime}\left(p_{1}^{\prime \prime}, p_{2}^{\prime \prime}, p_{3}^{\prime \prime}, p_{4}^{\prime \prime}\right) \cdot C^{\prime}\left(p_{2}^{\prime \prime}, p_{3}^{\prime \prime}, p_{4}^{\prime \prime}\right) .
\end{aligned}
$$

But $C^{\prime}\left(p_{1}^{\prime \prime}, p_{2}^{\prime \prime}, p_{3}^{\prime \prime}, p_{4}^{\prime \prime}\right)=\Lambda\left(p_{1}^{\prime}, p_{2}^{\prime}, p_{3}^{\prime}, p_{4}^{\prime}\right)=\Lambda\left(\alpha_{0}\right)=0$, and $C^{\prime}\left(p_{2}^{\prime \prime}, p_{3}^{\prime \prime}, p_{4}^{\prime \prime}\right)$ and $D^{\prime}\left(p_{1}^{\prime \prime}, p_{2}^{\prime \prime}, p_{3}^{\prime \prime}, p_{4}^{\prime \prime}\right)$ are both positive and we are thus led to a contradiction. Therefore, $\Lambda\left(\frac{1}{2}\right)<0$ and the theorem is proved for $\alpha=\frac{1}{2}$.

Since the above argument shows that $\Lambda(\alpha)$ cannot vanish for any value of $\alpha$ between zero and $\frac{1}{2}$, it follows that $\Lambda(\alpha)<0$ for $0<\alpha<\frac{1}{2}$. Hence 
the elements $p_{i}^{\prime}(i=1,2,3,4)$ of $\phi(M)$ with

$$
p_{i}^{\prime} p_{j}^{\prime}=\cosh ^{-1}\left[\left(p_{i} p_{j}\right)^{2 \alpha}+1\right] \quad(i, j=1,2,3,4), 0<\alpha<\frac{1}{2},
$$

are congruent with four points of three-dimensional hyperbolic space, and the theorem is proved.

We note that $\alpha=\frac{1}{2}$ is the greatest exponent for which the above theorem is valid. For example, let the points $p_{i}(i=1,2,3,4)$ form a pseudolinear quadruple with

$$
p_{1} p_{2}=p_{2} p_{3}=p_{3} p_{4}=p_{1} p_{4}=1, \quad p_{1} p_{3}=p_{2} p_{4}=2 \text {. }
$$

Now if this set is transformed by $\phi(x)=\cosh ^{-1}\left[x^{2([1 / 2]+\varepsilon)}+1\right]$ we find that

$$
\Lambda\left(p_{1}^{\prime}, p_{2}^{\prime}, p_{3}^{\prime}, p_{4}^{\prime}\right)=\left[4+2 \cdot 2^{\varepsilon}\right]\left[-2 \cdot 2^{2 \varepsilon}\right]\left[1-\left(2 \cdot 2^{2 \varepsilon}-1\right)^{2}\right]
$$

which vanishes for $\varepsilon=0$ and is positive for $\varepsilon>0$. Hence $p_{1}^{\prime}, p_{2}^{\prime}, p_{3}^{\prime}, p_{4}^{\prime}$ are congruent with four points of the hyperbolic plane for $\varepsilon=0$, while if $\varepsilon>0$, the four points are not congruently imbeddable in any hyperbolic space of curvature -1 .

\section{REFERENCES}

1. L. M. Blumenthal, Theory and applications of distance geometry, Clarendon Press, Oxford, 1953. MR 14, 1009.

2. - Remarks concerning the Euclidean four-point property, Ergebnisse Math. Kolloq. Wien 7 (1936), 7-10.

3. - The geometry of a class of semimetric spaces, Tôhoku Math. J. 43 (1937), 205-224.

4. I. J. Schoenberg, Metric spaces and positive definite functions, Trans. Amer. Math. Soc. 44 (1938), 522-536.

5. - On certain metric spaces arising from Euclidean spaces by a change of metric and their imbedding in Hilbert space, Ann. of Math. 38 (1937), 787-793.

6. - Metric spaces and completely monotone functions, Ann. of Math. 39 (1938), 811-841.

Department of Mathematics, Utah State University, Logan, Utah 84321 